\title{
Diagnosis of Traumatic Pneumothorax: A Comparison between Lung Ultrasound and Supine Chest Radiographs
}

\author{
Rohit Bhoil ${ }^{1} \odot$, Ranesh Kumar², Jaswinder Kaur ${ }^{3}$, Pardeep K Attri ${ }^{4}$, Rohini Thakur ${ }^{5}$
}

\begin{abstract}
Background/Objective: Traumatic pneumothorax is an ominous condition necessitating urgent appropriate action. It is typically detected on chest X-rays; however, these may not be able to detect the presence of a subtle pneumothorax, especially in supine position. Lung ultrasound is emerging as a promising modality for detecting pneumothorax in trauma patients. The aim of our study was to compare ultrasound with supine chest radiography for the detection of pneumothorax in trauma patients.

Materials and Methods: This was a prospective, single-blinded study carried out on 212 adult thoracoabdominal trauma patients who underwent ultrasound FAST and supine (AP) chest radiography. During the FAST sonography, ultrasound thorax was done to rule out pneumothorax. Only those cases were considered (118) in which the presence or absence of pneumothorax could be confirmed on CT done subsequently or where pneumothorax was confirmed by air escape on chest tube placement, wherever indicated, and the results were compared with sonographic and chest X-ray findings.

Observation/Results: There were 48 true positives on $\mathrm{CT} / \mathrm{chest}$ tube insertion. Among these, ultrasound was able to correctly detect pneumothorax in 43 patients, while supine chest X-rays correctly identified 33 cases. Sensitivity of ultrasound was 89.6 vs. $68.8 \%$ of supine chest radiography. Lung ultrasound also had a higher negative predictive value as compared to supine chest X-rays.

Conclusions: Lung ultrasound is more sensitive in detecting traumatic pneumothorax than supine chest X-rays, in addition to having numerous other inherent advantages over chest radiography. It should be incorporated in the emergency assessment of thoracic trauma patients to rule out pneumothorax.

Clinical significance: Lung sonography is more sensitive in detecting traumatic pneumothorax than supine chest X-rays. No added equipment is required, and the procedure can be carried out at the time of doing ultrasound FAST, thus saving precious time in trauma patients.

Keywords: Chest X-ray, Mortality, Thorax trauma, Ultrasound.

Indian Journal of Critical Care Medicine (2021): 10.5005/jp-journals-10071-23729
\end{abstract}

\section{INTRODUCTION}

Pneumothorax is an ominous condition, which may occur in thoracic injuries. It occurs when either or both of visceral and/ or parietal pleura ruptures allowing entry of air into the pleural space. ${ }^{1,2}$ Traumatic pneumothorax may be encountered in either penetrating (like stab injury, gunshot wound) or blunt chest trauma leading to rib fractures. ${ }^{2,3}$ Regardless of the cause, its timely and accurate detection and immediate management are crucial to prevent catastrophic consequences. ${ }^{4}$

Typically, in thoracic trauma patients, plain chest radiographs are done to look for pneumothorax. However, due to associated spinal and other injuries, radiographs are obtained in supine position (AP-anteroposterior view) in which typical features of pneumothorax may not be always clear. ${ }^{5,6}$ It has been reported in the literature that in trauma patients, subtle pneumothorax may be missed on initial clinical examination or on radiographs done at the time of presentation in up to 30 to $50 \%$ of cases. ${ }^{7}$

Thoracic computed tomography (CT) is the established gold standard investigation for the diagnosis of pneumothorax. ${ }^{7,8}$ However, its use on routine basis is limited by the fact that it involves high amount of exposure to harmful ionizing radiations; the patient needs to be transported for CT scanning, which may not be feasible in unstable patients. Moreover, it is expensive and may not be available in all centers, especially in less developed countries.

Ultrasound of the lung is emerging as a promising modality for detecting pneumothorax in critically ill and in trauma patients. ${ }^{9-13}$
1,4,5 Department of Radiodiagnosis, SLBS Medical College, Mandi, Himachal Pradesh, India

${ }^{2}$ Department of Surgery, SLBS Medical College, Mandi, Himachal Pradesh, India

${ }^{3}$ Department of Community Medicine, SLBS Medical College, Mandi, Himachal Pradesh, India

Corresponding Author: Rohit Bhoil, Department of Radiodiagnosis, SLBS Medical College, Mandi, Himachal Pradesh, India, Phone: +91 2147483647, e-mail: rohitbhoil@gmail.com

How to cite this article: Bhoil R, Kumar R, Kaur J, Attri PK, Thakur R. Diagnosis of Traumatic Pneumothorax: A Comparison between Lung Ultrasound and Supine Chest Radiographs. Indian J Crit Care Med 2021;25(2):176-180.

Source of support: Nil

Conflict of interest: None

It has numerous advantages when compared to chest radiography and $C T$ scanning, which includes not using ionizing radiations, being portable, capable of real-time imaging, and ideally suited for repeat examinations. Additionally, it is widely available and is not expensive.

We carried out a studyto evaluate and compare ultrasound with supine (anteroposterior) chest radiography for the detection of pneumothorax in trauma patients. 


\section{Materials and Methods}

This was a prospective, single-blinded study performed over a period of 19 months.

\section{Criteria of Selection and Exclusion Criteria}

Patients with thoracic or thoracoabdominal trauma, who underwent ultrasound FAST (focused abdominal sonography in trauma) ${ }^{14}$ and supine chest radiography were included in the study). At the time of doing FAST ultrasound, sonography of the thorax was performed to rule out pneumothorax, and the results were recorded. Supine chest radiographs were done, immediately after the ultrasound examination, and the $\mathrm{X}$-ray films were read by the attending trauma physician/surgeon, who was blinded to the results of the ultrasound lung findings.

Only those cases were considered in which $\mathrm{CT}$ scan of the thorax was done within 3 hours of the sonographic/radiographic evaluation. The presence/absence of pneumothorax on such CT scans was taken as the gold standard and used to compare with sonographic and radiographic findings. Cases in which the presence of pneumothorax could be confirmed by air escape upon chest tube placement (wherever done based on clinical urgency) were also taken as positive, irrespective of whether CT was subsequently done or not.

Patients less than 18 years of age were excluded from the study.

\section{Number of Patients}

Of the 212 adult thoracic or thoracoabdominal trauma patients who underwent supine chest radiography and ultrasound FAST (and hence ultrasound of the lungs), thoracic CT was performed in 169 cases. However, as we only considered cases in which CT was done within 3 hours of the sonographic/radiographic examination, 118 cases were finally selected (this figure included 11 cases in which the presence of pneumothorax was confirmed by air escape on chest tube placement).

\section{Ultrasound Thorax Procedure to Detect Pneumothorax}

All the sonographic examinations were performed on a SonoSite M-Turbo (Bothell, WA, USA) portable ultrasound machine, using a 6 to $15 \mathrm{MHz}$ linear probe. Protocol consisted of scanning at four different sites in each hemithorax, i.e., second intercostal space (along the midclavicular line), fourth intercostal space (along the anterior axillary line), sixth intercostal space (along the midaxillary line), and sixth intercostal space (along the posterior axillary line). Scanning was done with the probe in longitudinal position, perpendicular to the skin.

In normal lung, with respiration, a "sliding sign" is seen, which is due to the sliding movement of visceral and parietal pleura (seen as bright or echogenic line on ultrasound) past each other (Fig. 1A; Supplementary Video S1). This corresponds to "seashore" sign on activating the M-mode of ultrasound (Fig. 1B). ${ }^{15-17}$ In case of pneumothorax, sliding sign is absent and a "barcode" sign or "stratosphere" sign (Fig. 2) is seen on M-mode instead of the normal seashore sign, which is due to the presence of air in between the two pleural layers. ${ }^{18}$

The absence of lung sliding sign and seashore sign and presence of barcode sign at the same location were diagnosed as the presence of pneumothorax on sonographic evaluation. All sonographic procedures were done by any of the three radiologists on duty.

Written informed consent was obtained from each patient or his/her nearest attendant, who was included in the study.

\section{Statistical Analysis}

We used Statistical Package for Social Sciences (SPSS) 16.0 for Windows for the statistical analysis of the data obtained. $95 \%$ confidence interval was considered. Sensitivity, specificity, positive and negative predictive values, and likelihood ratios were calculated by comparing the results of thoracic sonography and supine chest radiographs with the gold standard (CT or air escape on chest tube insertion, wherever done).

\section{Observations and Results}

The study comprised 118 patients (age range of 18-68 years; mean age 46.8 years; M:F:: 74:38) who underwent all the investigative procedures, viz. (1) thoracic ultrasound, (2) supine chest radiograph, and (3) thoracic CT within 3 hours of the sonographic/radiographic evaluation or presence of air escape on chest tube insertion, wherever done (due to clinical urgency).

Out of these 118 selected cases, true positives on CT/chest tube insertion were 48 patients (40.68\%): 37 positive on CT and 11 having air escape on chest tube insertion. Among these, ultrasound was
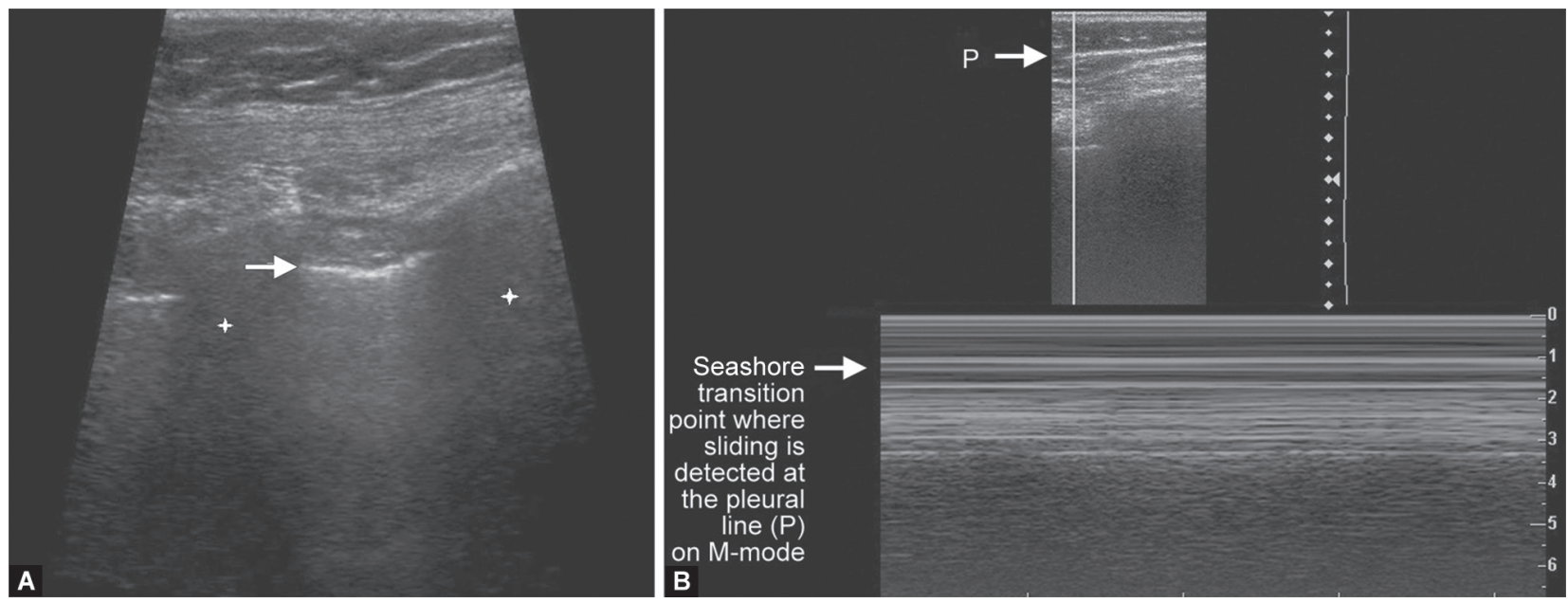

Figs. $1 \mathrm{~A}$ and B:Thoracic ultrasound images (using a 6-15 MHz linear probe) in a normal lung showing (A) the pleural line seen as a bright echogenic line (arrow); stars represent the adjoining rib shadows; and (B) M-mode ultrasound image showing the "seashore" sign 


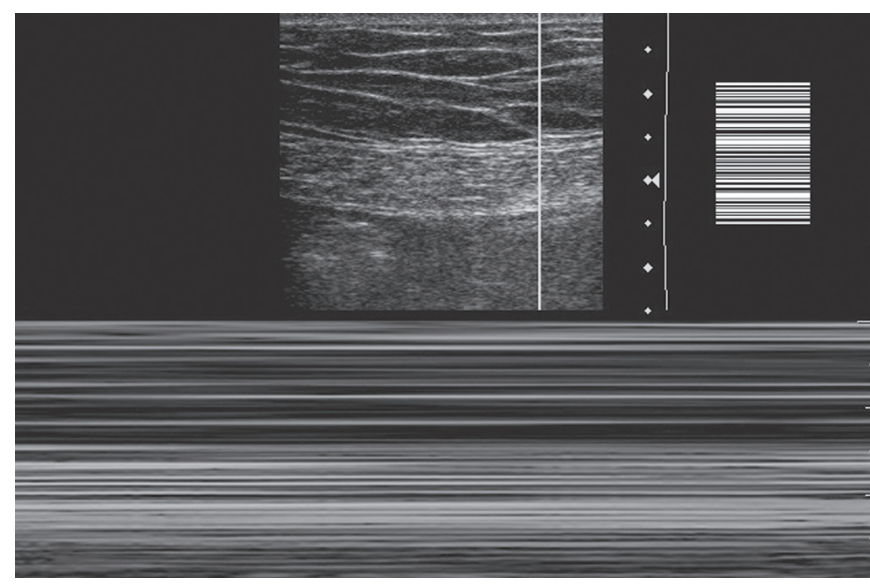

Fig. 2: Thoracic ultrasound image (using a 6-15 MHz linear probe) in the presence of pneumothorax showing the "stratosphere" or "barcode" sign on M-mode ultrasound

Table 1A: Lung ultrasound and CT cross-tabulation data

\begin{tabular}{lllll}
\hline & & \multicolumn{2}{c}{$C T$} & \\
\cline { 3 - 5 } & & $Y^{*}$ & $N$ & Total \\
\hline \multirow{2}{*}{ USG } & $\mathrm{Y}$ & 43 & 5 & 48 \\
& $\mathrm{~N}$ & 5 & 65 & 70 \\
Total & & 48 & 70 & 118 \\
\hline
\end{tabular}

*Includes 11 cases with air escape on chest tube insertion; USG, ultrasound thorax; $\mathrm{CT}$, computed tomography

Table1B: Supine chest X-ray and CT cross-tabulation data

\begin{tabular}{lllll}
\hline & & \multicolumn{2}{c}{$C T$} & \\
\cline { 3 - 4 } & & $Y^{*}$ & $N$ & Total \\
\hline CXR & $Y$ & 33 & 2 & 35 \\
& $N$ & 15 & 68 & 83 \\
Total & & 48 & 70 & 118
\end{tabular}

*Includes 11 cases with air escape on chest tube insertion; USG, ultrasound thorax; $\mathrm{CT}$, computed tomography

able to correctly detect pneumothorax in 43 patients. However, only 33 patients were correctly identified on supine chest radiographs.

Tables $1 \mathrm{~A}$ and $1 \mathrm{~B}$ depict the results of our study.

Overall, the sensitivity to detect pneumothorax in trauma patients was much higher in lung ultrasound as compared to chest radiographs ( 89.58 vs. $68.75 \%$ ). The negative predictive value of ultrasound was also more than that of supine chest X-ray. Table 2 shows the sensitivities, specificities, positive predictive values (PPVs), negative predictive values (NPVs), and likelihood ratios for US and supine chest radiography.

Each site was scanned for about 10 to 15 seconds, thus completing the sonographic examination of entire thorax in about 2 minutes.

\section{Discussion}

Pneumothorax is commonly associated with trauma to thoracic and/or thoracoabdominal regions. Its timely detection and management are important to prevent subsequent morbidity and mortality in such patients. Traditionally, plain chest radiographs
Table 2: Results of various statistical parameters calculated in the study

\begin{tabular}{lll}
\hline & USG & CXR \\
\hline Sensitivity & $89.58 \%$ & $68.75 \%$ \\
Specificity & $95.59 \%$ & $97.14 \%$ \\
PPV & $89.58 \%$ & $94.29 \%$ \\
NPV & $95.59 \%$ & $81.92 \%$ \\
LR+ & 20.31 & 24.04 \\
LR- & 0.11 & 0.32 \\
\hline
\end{tabular}

PPV, positive predictive value; NPV, negative predictive value; $\mathrm{LR}+$, positive likelihood ratio; LR-, negative likelihood ratio

have been used as the initial screening tool for the detection of pneumothorax in trauma patients. However, these are not very sensitive in correctly identifying cases of subtle pneumothorax; this is especially true in case of trauma patients where due to associated spinal injuries and other bodily injuries it may not be possible to take the radiograph in erect position, which necessitates that the radiographs are obtained in supine position (AP-anteroposterior view) in which typical features of pneumothorax may not be always present. ${ }^{5,6}$ Kirkpatrick et al. ${ }^{7}$ in a study on 225 trauma patients demonstrated that an AP supine chest radiograph has a sensitivity of $20.9 \%$ for detecting pneumothorax when compared with results on thoracic CT scans.

Ultrasonographic evaluation of the chest has been used in emergency and critical care setting since long and is well established..$^{18-18} \mathrm{It}$ is now being increasingly used in the evaluation of acute respiratory emergencies including but not limited to pneumothorax..$^{18}$ Ultrasound of thorax has been reported in several studies as a reliable and much more accurate method in detecting traumatic pneumothorax. ${ }^{9-13}$ It has numerous advantages when compared to chest radiography and CT scanning, which includes not using ionizing radiations, being portable, capable of real-time imaging, and ideally suited for repeat examinations. Additionally, it is widely available and is not expensive.

In our study, we attempted to compare ultrasound with supine (anteroposterior) chest radiography for the detection of pneumothorax in trauma patients. Our study demonstrated that the lung ultrasound was a better imaging modality for the detection of traumatic pneumothorax as compared to supine chest radiographs, being more sensitive. This finding is in agreement with other such studies comparing the two modalities (lung ultrasound vs. chest radiography). This is especially true in cases of small pneumothoraces, which may be missed on radiographs.

Nagarsheth and Kurek $^{10}$ in 2011 carried out a prospective study on 79 trauma patients comparing thoracic ultrasound with chest radiograph and CT scan in the detection of traumatic pneumothorax. The study comprised of 22 confirmed cases of pneumothorax on CT scans. They reported sensitivity of $81.8 \%$ for thoracic ultrasound and only $31.8 \%$ for chest radiograph. The specificity was $100 \%$ for both thoracic ultrasound and chest X-ray.

Wilkerson and Stone ${ }^{11}$ carried out a review of trials conducted between 1965 and 2009 comparing sensitivity of bedside US and AP chest radiographs in identifying pneumothorax after blunt trauma. They reported the sensitivity of thoracic ultrasound that ranged from 86 to $98 \%$, which was more than that of supine chest $X$-rays that ranged between 28 and $75 \%$. However, both had similar specificities ranging between 97 and 100\% for ultrasound and 100\% for chest radiograph. 
It is worth mentioning here that though thoracic ultrasound is more sensitive than chest radiograph in detecting traumatic pneumothorax, its use largely depends on the operator's experience. Although there is a learning curve associated with the use of chest US, it is relatively short. In a meta-analysis ${ }^{12}$ conducted in 2011, evaluating all articles published in English medical literature that used either ultrasound or chest radiograph or both to diagnose a pneumothorax, the pooled sensitivity and specificity of ultrasound performed were 89 and $99 \%$, respectively, whereas the sensitivity and specificity of chest radiographs were only 52 and $100 \%$, respectively. They reported similar results in both trauma patients and mechanically ventilated critically ill patients. The authors stressed that the accuracy of ultrasonography in the diagnosis of pneumothorax depended on the skill of the operators.

In our study, all sonographic examinations were conducted by one of the three radiologists, each having more than 4 years of experience in sonography including emergency ultrasounds. Further, in order to ensure that the lung fields were adequately scanned, we examined each hemithorax at four different preset points in each patient.

These data support the use of transthoracic ultrasound as the initial screening tool for pneumothorax diagnosis. Although the specificity of chest radiograph and bedside ultrasound is essentially equivalent, an ultrasound is much more sensitive than chest radiograph for detecting a pneumothorax. In addition, the diagnostic characteristics of a transthoracic ultrasound compare favorably with a chest CT scan with much less cost, time, radiation exposure, and no need for patient transport.

Blaivas $^{13}$ also reported that lung ultrasound is more sensitive in diagnosing traumatic pneumothorax than plain AP chest radiography. They used a $3.5 \mathrm{MHz}$ microconvex transducer, which is also used for doing ultrasound FAST examinations. They concluded that the use of a microconvex probe has the added advantage of being adequate for performing both examinations. However, in our study, we used high-frequency probe $(6-15 \mathrm{MHz})$, which provided a better imaging quality and allowed for easy detection of lung sliding, visualization of which effectively rules out pneumothorax. In doubtful or in difficult cases, like patient obesity, M-mode was especially useful in visualizing the seashore sign in normal cases, which was replaced by the barcode sign in cases of pneumothorax. Similar observations have been made by other researchers as well. ${ }^{18}$

In addition to the obvious advantage of lung ultrasound being more sensitive as compared to chest radiographs (A/P view), the authors are of the opinion that the use of thoracic sonography has the potential to save precious time in trauma patients; the procedure can be done at the time of doing ultrasound FAST examination, and no other specialized equipment is required. Moreover, no harmful radiations are used and it is cheaper.

One of the limitations of our study was that not all patients who underwent CT could be included as we only considered cases that underwent CT examination within 3 hours of sonographic/ radiographic evaluation. However, inspite of these criteria, some of the false negatives on ultrasound and/or chest X-rays could have been due to the enlargement of pneumothorax (due to air leak) in CT done subsequently. Similarly, few of the false positives on ultrasound and/or chest X-rays could have been due to spontaneous closure/sealing of traumatic pneumothorax in CT done subsequently. It is to be noted that ultrasound of the thorax may be suboptimal in certain cases like subcutaneous emphysema or flail chest, though none of the cases in our study had these conditions.
Another limitation of our study was that we did not quantify the extent of pneumothorax or grade it according to severity (mild/moderate/massive), which would have increased its value in comparing the two modalities.

\section{Conclusion}

Lung sonography is more sensitive in detecting traumatic pneumothorax than supine chest X-rays. No added equipment is required, and the procedure can be carried out at the time of doing ultrasound FAST, thus saving precious time in trauma patients. Moreover, unlike radiography, no ionizing radiations are used in sonography. Keeping in view these observations, the authors conclude that lung ultrasound should be incorporated in the emergency assessment of thoracic trauma patients to confirm the presence or absence of pneumothorax, especially when erect radiographs cannot be obtained due to associated spinal and other injuries.

\section{Compliance with Ethical Standards}

Statement of human and animal rights: All procedures performed instudies involving human participants were in accordance with the 1964 Helsinki Declaration and its later amendments or comparable ethicalstandards.

Written informed consent was obtained from each patient or his/her nearest attendant, who was included in the study.

\section{OrCID}

Rohit Bhoil @ https://orcid.org/0000-0002-5155-8325

\section{References}

1. Hefny AF, Kunhivalappil FT, Matev N, Avila NA, Bashir MO, Abu-Zidan FM. Management of computed tomography-detected pneumothorax in patients with blunt trauma: experience from a community-based hospital. Singapore Med J 2018;59(3):150-154. DOI: 10.11622/smedj.2017074.

2. Zarogoulidis P, Kioumis I, Pitsiou G, Porpodis K, Lampaki S, Papaiwannou $A$, et al. Pneumothorax: from definition to diagnosis and treatment. J Thorac Dis 2014;6(Suppl. 4):S372-S376. DOI: 10.3978/j.issn.2072-1439.2014.09.24.

3. Kaneda H, Nakano T, Taniguchi Y, Saito T, Konobu T, Saito Y. Threestep management of pneumothorax: time for a re-think on initial management. Interact Cardiovasc Thorac Surg 2013;16(2):186-192. DOI: 10.1093/icvts/ivs445.

4. Ince A, OzucelikDN, Avci A, Nizam O, Dogan H, Topal MA. Management of pneumothorax in emergency medicine departments: multicenter trial. Iran Red Crescent Med J 2013;15(12):e11586. DOI: 10.5812/ ircmj.11586.

5. Ball CG, Kirkpatrick AW, Laupland KB, Fox DL, Litvinchuk S, Dyer DM, et al. Factors related to the failure of radiographic recognition of occult posttraumatic pneumothoraces. Am J Surg 2005;189(5):541546. DOI: 10.1016/j.amjsurg.2005.01.018.

6. Lewis FR, Blaisdell FW, Schlobohm RM. Incidence and outcome of posttraumatic respiratory failure. Arch Surg 1977;112(4):436-443. DOI: 10.1001/archsurg.1977.01370040088014.

7. Kirkpatrick AW, Sirois M, Laupland KB, Liu D, Rowan K, Ball CG, et al. Hand-held thoracic sonography for detecting post-traumatic pneumothoraces: the extended focused assessment with sonography for trauma (EFAST). J Trauma 2004;57(2):288-295. DOI: 10.1097/01.ta.0000133565.88871.e4.

8. Husain LF, Hagopian L, Wayman D, Baker WE, Carmody KA. Sonographic diagnosis of pneumothorax. J Emerg Trauma Shock 2012;5(1):76-81. DOI: 10.4103/0974-2700.93116. 
9. Ebrahimi A, Yousefifard M, Mohammad Kazemi H, Rasouli HR, Asady H, Moghadas Jafari A, et al. Diagnostic accuracy of chest ultrasonography versus chest radiography for identification of pneumothorax: a systematic review and meta-analysis. Tanaffos 2014;13(4):29-40.

10. Nagarsheth K, Kurek S. Ultrasound detection of pneumothorax compared with chest X-ray and computed tomography scan. Am Surg 2011;77(4):480-484.

11. Wilkerson RG, Stone MB. Sensitivity of bedside ultrasound and supine anteroposterior chest radiographs for the identification of pneumothorax after blunt trauma. Acad Emerg Med 2010;17(1):11-17. DOI: 10.1111/j.1553-2712.2009.00628.x.

12. Ding W, Shen $Y$, Yang J, He X, Zhang M. Diagnosis of pneumothorax by radiography and ultrasonography: a meta-analysis. Chest 2011;140(4):859-866. DOI: 10.1378/chest.10-2946.

13. Blaivas $M$, Lyon $M$, Duggal $S$. A prospective comparison of supine chest radiography and bedside ultrasound for the diagnosis of traumatic pneumothorax. Acad Emerg Med 2005;12(9):844-849. DOI: 10.1197/j.aem.2005.05.005.

14. Chaudhry R, Galagali A, Narayanan RV. Focused abdominal sonography in trauma (FAST). Med J Armed Forces India 2007;63(1): 62-63. DOI: 10.1016/S0377-1237(07)80113-4.

15. Lee FC. Lung ultrasound-a primary survey of the acutely dyspneic patient. J Intensive Care 2016;4(1):57. DOI: 10.1186/s40560-016-0180-1.

16. Saraogi A. Lung ultrasound: present and future. Lung India 2015;32(3):250-257. DOI: 10.4103/0970-2113.156245.

17. Lichtenstein D. Novel approaches to ultrasonography of the lung and pleural space: where are we now? Breathe (Sheff) 2017;13(2):100-111. DOI: $10.1183 / 20734735.004717$

18. Abdalla W, Elgendy M, Abdelaziz AA, Ammar MA. Lung ultrasound versus chest radiography for the diagnosis of pneumothorax in critically ill patients: a prospective, single-blind study. Saudi J Anaesth 2016;10(3):265-269. DOI: 10.4103/1658-354X.174906.

Supplementary Video S1:Video showing the sliding sign, seen in normal lung due to the sliding movement of visceral and parietal pleura (seen as bright or echogenic line on ultrasound) past each other 